\title{
Plane-Wave Characterization of Antennas Close to a Planar Interface
}

\author{
Meincke, Peter; Hansen, Thorkild
}

Published in:

I E E E Transactions on Geoscience and Remote Sensing

Link to article, DOI:

10.1109/TGRS.2004.825590

Publication date:

2004

Document Version

Publisher's PDF, also known as Version of record

Link back to DTU Orbit

Citation (APA):

Meincke, P., \& Hansen, T. (2004). Plane-Wave Characterization of Antennas Close to a Planar Interface. I E E E Transactions on Geoscience and Remote Sensing, 42(6), 1222-1232.

https://doi.org/10.1109/TGRS.2004.825590

\section{General rights}

Copyright and moral rights for the publications made accessible in the public portal are retained by the authors and/or other copyright owners and it is a condition of accessing publications that users recognise and abide by the legal requirements associated with these rights.

- Users may download and print one copy of any publication from the public portal for the purpose of private study or research.

- You may not further distribute the material or use it for any profit-making activity or commercial gain

- You may freely distribute the URL identifying the publication in the public portal

If you believe that this document breaches copyright please contact us providing details, and we will remove access to the work immediately and investigate your claim 


\title{
Plane-Wave Characterization of Antennas Close to a Planar Interface
}

\author{
Peter Meincke, Member, IEEE, and Thorkild B. Hansen, Member, IEEE
}

\begin{abstract}
The plane-wave scattering matrix is used to characterize antennas that are located just above a planar interface that separates two media. The plane-wave transmitting spectrum for the field radiated downward into the lower medium is expressed directly in terms of the current distribution of the transmitting antenna. The transmitting spectrum for a reciprocal antenna determines the plane-wave receiving spectrum for the field that propagates upward in the lower medium. A measurement procedure is discussed for determining the plane-wave transmitting and receiving spectra from measurements with a probe that is located in the lower medium.
\end{abstract}

Index Terms-Ground-penetrating radar (GPR), planar interface, planar near-field measurements, plane-wave scattering matrix, subsurface imaging, surface-penetrating radar (SPR).

\section{INTRODUCTION}

$\mathbf{T}$ HE PLANE-WAVE scattering matrix of an antenna-comprising the reflection coefficient and the plane-wave transmitting, receiving, and scattering spectra-is widely used for characterizing antennas [1] and plays an important role in conventional planar near-field antenna measurements [1], [2]. With such measurements, the antenna is in a free-space environment where multiple interactions between the antenna and nearby objects can be neglected. Hence, the scattering spectrum is omitted from the formulation.

In other important applications, however, the scattering spectrum cannot be neglected because strong multiple interactions exist between the environment and the antenna. Indeed, multiple interaction are critically important in determining the properties of antennas that are located in free space just above a planar interface (throughout the paper, we use the term "antenna under test" (AUT) to describe an antenna that is located in free space close to a planar interface). Such antennas appear frequently in for instance surface- and ground-penetrating radar (SPR/GPR) applications [3]-[5]. For the AUT, the quantities of interest relate the voltage at the antenna terminals and the fields in the lower medium. These quantities can in principle be determined from the free-space plane-wave scattering matrix of the antenna [6]. Since strong multiple interactions exist between the antenna and the interface, the free-space scattering spectrum cannot be omitted in this case. Further, because the AUT is close to the interface, one must not only include the components of the scattering spectrum that determine the interaction with propagating

Manuscript received January 28, 2003; revised January 13, 2004. The work of P. Meincke was supported by the Danish Technical Research Council.

P. Meincke is with Ørsted-DTU, Electromagnetic Systems, Technical University of Denmark, DK-2800 Lyngby, Denmark (e-mail: pme@ oersted.dtu.dk).

T. B. Hansen is with Seknion, Inc., Boston, MA 01235 USA (e-mail: thorkild.hansen@worldnet.att.net).

Digital Object Identifier 10.1109/TGRS.2004.825590 waves, but also a part of the components that determine the interaction with evanescent waves. Since the free-space scattering spectrum is very difficult to determine for realistic antennas (especially the part that accounts for the interaction with evanescent waves), we shall not use the free-space scattering matrix formulation to characterize the AUT.

Fortunately, one can avoid using the free-space scattering spectrum for the AUT because the antenna interaction with the fields in the lower medium can be described by a "total" scattering matrix that takes into account the multiple interactions at the interface. By "the scattering matrix for the AUT" we mean the scattering matrix for the system consisting of the antenna and the planar interface. This scattering matrix-i.e., the reflection coefficient, the transmitting spectrum, the receiving spectrum, and the scattering spectrum-relates the voltage at the antenna terminals to the outgoing (downward propagating) and incoming (upward propagating) fields in the lower medium. The scattering matrix constitutes an exact representation of both near- and far fields radiated, received, and scattered by the AUT/interface system. The effect of the multiple interactions between the interface and the antenna does not appear explicitly in the formulation, but are accounted for by the scattering matrix. As a consequence, the scattering matrix for the AUT depends on the antenna itself, the electromagnetic properties of the lower medium, and the distance between the antenna and the interface.

In practice the scattering matrix for the AUT can be determined by first computing the current distribution of the antenna when it is situated above the planar interface. This computation can be performed with numerical procedures such as the method of moments [7], [8] and the finite-difference time-domain method [9]. The resulting currents can be used to determine the desired transmitting and receiving spectra. For certain simple antennas, explicit expressions exist for the current distribution [10, Sec. 1.9]. Alternatively, the spectra can be determined from measurements with a probe located in the lower medium [11].

The paper is organized as follows. We briefly discuss the freespace scattering matrix in Section II and define the scattering matrix for the AUT in Section III. In Section IV, we derive a relation between the transmitting spectrum and the current density of the transmitting AUT. A formula for the receiving spectrum for a reciprocal AUT in terms of the transmitting spectrum is derived in Section V. In Section VI, we describe a measurement procedure for determining the transmitting and receiving spectra with a fixed probe that is placed in the lower medium. The transmitting spectrum is determined by measuring two orthogonal components of the electric field parallel to the interface 


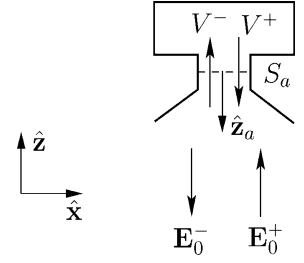

Fig. 1. Free-space antenna attached to a coaxial cable.

with the probe for each position of the AUT on a grid parallel to the interface. By neglecting multiple interactions between the AUT/interface system and the probe, i.e., by omitting the scattering spectrum from the formulation, the desired transmitting spectrum can be determined directly by Fourier transforming the output of the probe with respect to the antenna position. Multiple interactions between the probe and the AUT/interface system are typically negligible because of the losses in the lower medium. A similar procedure is described for measuring the receiving spectrum. Finally, in Section VII, numerical simulations illustrate some of the considerations involved in measuring the plane-wave spectra of the AUT.

Throughout the paper, the time factor $\exp (-i \omega t)$ is assumed and suppressed.

\section{Free-Space ScAttering Matrix}

Fig. 1 shows an antenna that is located in free space with permittivity $\epsilon_{0}$, permeability $\mu_{0}$, and wavenumber $k_{0}=\omega \sqrt{\mu_{0} \epsilon_{0}}$. The position of the antenna is given by $x=x_{a}, y=y_{a}$, and $z=$ $z_{a}$ in the usual rectangular $x y z$ coordinate system. The antenna is attached to a coaxial cable with inner radius $a$, outer radius $b$, and characteristic admittance $Y_{c}=2 \pi(\ln (b / a))^{-1} \sqrt{\epsilon / \mu}$, with $\epsilon$ and $\mu$ denoting the permittivity and permeability, respectively, of the dielectric material separating the conductors. It is assumed that just one propagating transverse electromagnetic (TEM) mode is excited in the cable. In the reference plane $S_{a}$ of the antenna, shown in Fig. 2, the total field is the sum of contributions from an incident field, propagating in the cable toward the antenna, and an emerging field, propagating away from the antenna. Hence, in the reference plane, the voltage $V$ between the inner and outer conductors can be written as $V=V^{+}+V^{-}$ where the superscript + refers to the incident field and the superscript - to the emerging field. The voltage can also be written as $V=V^{+}(1+\Gamma)$, where $\Gamma$ is the reflection coefficient at the reference plane seen toward the antenna. Similarly, in the reference plane, the current $I$ flowing in the inner conductor can be expressed as $I=I^{+}-I^{-}=\left(V^{+}-V^{-}\right) Y_{c}=V Y_{a}$ where $Y_{a}$ is the antenna admittance. The current $I$ is assumed positive when flowing into the antenna and negative when flowing out. Using the local polar $\rho_{a} \phi_{a} z_{a}$ coordinate system in Fig. 2, the electric and magnetic fields in the reference plane $S_{a}$ are [12, p. 218]

and

$$
\mathbf{E}=\frac{V \hat{\boldsymbol{\rho}}_{a}}{\rho_{a} \ln \frac{b}{a}}
$$

$$
\mathbf{H}=\frac{I \hat{\boldsymbol{\phi}}_{a}}{2 \pi \rho_{a}}
$$

respectively.

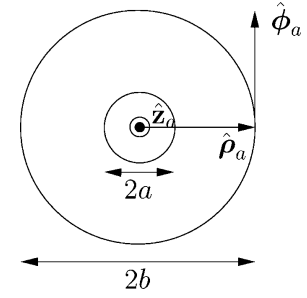

Fig. 2. Reference plane $S_{a}$ in the coaxial cable with inner radius $a$ and outer radius $b$.

The emerging field in the coaxial cable with voltage $V^{-}$in the reference plane is a sum of two contributions caused by the incident field with voltage $V^{+}$in the reference plane and the upward-propagating electric field $\mathbf{E}_{0}^{+}$, respectively (see Fig. 1). Similarly, the downward-propagating electric field $\mathbf{E}_{0}^{-}$is a sum of two contributions caused by the incident field with voltage $V^{+}$in the reference plane and the upward-propagating electric field $\mathbf{E}_{0}^{+}$, respectively. The downward- and upward-propagating electric fields are given by the following plane-wave expansions

$$
\begin{aligned}
& \mathbf{E}_{0}^{-}(x, y, z, \omega)=\frac{1}{(2 \pi)^{2}} \iint_{-\infty}^{\infty} \mathbf{S}_{0}^{-}\left(k_{x}, k_{y}, \omega\right) \\
& \cdot \exp \left(i\left[k_{x} x+k_{y} y-\gamma_{0} z\right]\right) d k_{x} d k_{y}
\end{aligned}
$$$$
\text { and }
$$

$$
\begin{aligned}
& \mathbf{E}_{0}^{+}(x, y, z, \omega)=\frac{1}{(2 \pi)^{2}} \iint_{-\infty}^{\infty} \mathbf{S}_{0}^{+}\left(k_{x}, k_{y}, \omega\right) \\
& \cdot \exp \left(i\left[k_{x} x+k_{y} y+\gamma_{0} z\right]\right) d k_{x} d k_{y}
\end{aligned}
$$

where $\gamma_{0}=\sqrt{k_{0}^{2}-k_{x}^{2}-k_{y}^{2}}$ with $\operatorname{Re} \gamma_{0} \geq 0$ and $\operatorname{Im} \gamma_{0} \geq 0$. Moreover, $\mathbf{S}_{0}^{-}$and $\mathbf{S}_{0}^{+}$are the plane-wave spectra of the downward- and upward-propagating electric field, respectively. The relations between the voltages and the fields are described through the free-space scattering matrix of the antenna, comprising the reflection coefficient $\Gamma_{f s}$, the transmitting spectrum $\mathbf{T}_{f s}$, the receiving spectrum $\mathbf{R}_{f s}$, and the scattering spectrum $\overline{\mathbf{S}}_{f s}$, as follows [1]:1

$$
\begin{aligned}
V^{-}(\omega)= & \Gamma_{f s}(\omega) V^{+}(\omega)+\frac{1}{(2 \pi)^{2}} \iint_{-\infty}^{\infty} \mathbf{R}_{f s}\left(k_{x}, k_{y}, \omega\right) \\
& \cdot \mathbf{S}_{0}^{+}\left(k_{x}, k_{y}, \omega\right) \exp \left(i\left[k_{x} x_{a}+k_{y} y_{a}+\gamma_{0} z_{a}\right]\right) d k_{x} d k_{y}
\end{aligned}
$$

and

$$
\begin{aligned}
\mathbf{S}_{0}^{-}\left(k_{x}, k_{y}, \omega\right)= & V^{+}(\omega) \mathbf{T}_{f s}\left(k_{x}, k_{y}, \omega\right) \\
& \cdot \exp \left(-i\left[k_{x} x_{a}+k_{y} y_{a}-\gamma_{0} z_{a}\right]\right) \\
& +\iint_{-\infty}^{\infty} \overline{\mathbf{S}}_{f s}\left(k_{x}, k_{y}, k_{x}^{\prime}, k_{y}^{\prime}, \omega\right) \cdot \mathbf{S}_{0}^{+}\left(k_{x}^{\prime}, k_{y}^{\prime}, \omega\right) \\
& \cdot \exp \left(i \left[\left(k_{x}^{\prime}-k_{x}\right) x_{a}+\left(k_{y}^{\prime}-k_{y}\right) y_{a}\right.\right. \\
& \left.\left.\quad+\left(\gamma_{0}+\gamma_{0}^{\prime}\right) z_{a}\right]\right) d k_{x}^{\prime} d k_{y}^{\prime}
\end{aligned}
$$

where $\gamma_{0}^{\prime}=\sqrt{k_{0}^{2}-k_{x}^{\prime 2}-k_{y}^{\prime 2}}$. Note that the transmitting and receiving spectra are vectors whereas the scattering spectrum is a $3 \times 3$ matrix. The receiving spectrum $\mathbf{R}_{f s}$ satisfies

\footnotetext{
${ }^{1}$ Kerns's plane-wave characterization of an antenna [1] was formulated in terms of transverse electric and magnetic field components. Here, we employ the formulation in [2] involving rectangular field components. This gives rise to a more simple notation.
} 


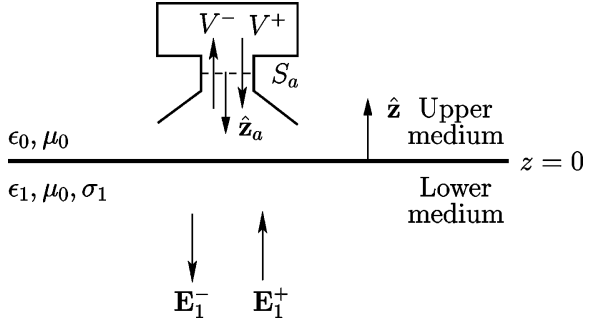

Fig. 3. Antenna close to a planar interface. The scattering matrix involves the plane-wave spectra of the upward- and downward-propagating electric field, $\mathbf{E}_{1}^{+}$and $\mathbf{E}_{1}^{-}$, respectively, in the lower medium.

$\mathbf{R}_{f s}\left(k_{x}, k_{y}, \omega\right) \cdot \mathbf{k}_{0}=0$, where $\mathbf{k}_{0}=k_{x} \hat{\mathbf{x}}+k_{y} \hat{\mathbf{y}}+\gamma_{0} \hat{\mathbf{z}}$, and the transmitting spectrum $\mathbf{T}_{f s}\left(k_{x}, k_{y}, \omega\right)$ satisfies $\mathbf{T}_{f s}\left(k_{x}, k_{y}, \omega\right) \cdot \mathbf{k}_{0}^{-}=0$, where $\mathbf{k}_{0}^{-}=k_{x} \hat{\mathbf{x}}+k_{y} \hat{\mathbf{y}}-\gamma_{0} \hat{\mathbf{z}}$. Throughout the paper, the operator $\cdot$ denotes the usual dot product, defined as $\mathbf{R}_{f s} \cdot \mathbf{k}_{0}=R_{f s x} k_{x}+R_{f s y} k_{y}+R_{f s z} \gamma_{0}$. If multiple interactions between the antenna and nearby objects can be neglected, the integral term of (6) is omitted.

\section{SCATTERING MATRIX FOR THE AUT}

Consider now the configuration in Fig. 3 where the antenna from Section II (now referred to as the AUT) is located close to a planar interface. The interface is at $z=0$, and the antenna is located in the upper medium, which is the region $z \geq 0$. The upper medium is assumed to have the same electromagnetic properties as free space. The region $z<0$ is denoted by the lower medium, and it possesses the permittivity $\epsilon_{1}$, permeability $\mu_{0}$, and conductivity $\sigma_{1}$. Hence, the wavenumber in the lower medium is $k_{1}=\sqrt{\omega^{2} \mu_{0} \epsilon_{1}+i \omega \mu_{0} \sigma_{1}}$. Except for Hertzian dipoles, and other antennas described by an impressed current density, strong multiple interactions exist between the antenna and the interface.

The current distribution of the antenna, and therefore all related antenna parameters, will be very different from the freespace case due to these multiple interactions. The interesting relations for the AUT relate the incident and emerging fields in the reference plane of the antenna to the downward- and upward-propagating electric fields in the lower medium. Indeed, the emerging field in the coaxial cable with voltage $V^{-}$in the reference plane is a sum of two contributions caused by the incident field with voltage $V^{+}$in the reference plane and the upward-propagating electric field $\mathbf{E}_{1}^{+}$in the lower medium, respectively. Similarly, the downward-propagating electric field $\mathbf{E}_{1}^{-}$in the lower medium is a sum of two contributions caused by the incident field with voltage $V^{+}$in the reference plane and the upward-propagating electric field $\mathbf{E}_{1}^{+}$in the lower medium, respectively. The electric fields in the lower medium are given by the plane-wave expansions

$$
\begin{array}{r}
\mathbf{E}_{1}^{-}(x, y, z, \omega)=\frac{1}{(2 \pi)^{2}} \iint_{-\infty}^{\infty} \mathbf{S}_{1}^{-}\left(k_{x}, k_{y}, \omega\right) \\
\cdot \exp \left(i\left[k_{x} x+k_{y} y-\gamma_{1} z\right]\right) d k_{x} d k_{y}
\end{array}
$$

and

$$
\begin{array}{r}
\mathbf{E}_{1}^{+}(x, y, z, \omega)=\frac{1}{(2 \pi)^{2}} \iint_{-\infty}^{\infty} \mathbf{S}_{1}^{+}\left(k_{x}, k_{y}, \omega\right) \\
\cdot \exp \left(i\left[k_{x} x+k_{y} y+\gamma_{1} z\right]\right) d k_{x} d k_{y}
\end{array}
$$

where $\gamma_{1}=\sqrt{k_{1}^{2}-k_{x}^{2}-k_{y}^{2}}$ with $\operatorname{Re} \gamma_{1} \geq 0$ and $\operatorname{Im} \gamma_{1} \geq 0$. Inspired by the free-space formulation, we now define the scattering matrix-i.e., the reflection coefficient $\Gamma$, the transmitting spectrum $\mathbf{T}$, the receiving spectrum $\mathbf{R}$, and the scattering spectrum $\overline{\mathbf{S}}$-for the AUT in the following way:

$$
\begin{aligned}
V^{-}(\omega)= & \Gamma(\omega) V^{+}(\omega)+\frac{1}{(2 \pi)^{2}} \iint_{-\infty}^{\infty} \mathbf{R}\left(k_{x}, k_{y}, \omega\right) \\
& \cdot \mathbf{S}_{1}^{+}\left(k_{x}, k_{y}, \omega\right) \exp \left(i\left[k_{x} x_{a}+k_{y} y_{a}\right]\right) d k_{x} d k_{y}
\end{aligned}
$$

and

$$
\begin{aligned}
\mathbf{S}_{1}^{-}\left(k_{x}, k_{y}, \omega\right)= & V^{+}(\omega) \mathbf{T}\left(k_{x}, k_{y}, \omega\right) \exp \left(-i\left[k_{x} x_{a}+k_{y} y_{a}\right]\right) \\
& +\iint_{-\infty}^{\infty} \overline{\mathbf{S}}\left(k_{x}, k_{y}, k_{x}^{\prime}, k_{y}^{\prime}, \omega\right) \cdot \mathbf{S}_{1}^{+}\left(k_{x}^{\prime}, k_{y}^{\prime}, \omega\right) \\
& \cdot \exp \left(i\left[\left(k_{x}^{\prime}-k_{x}\right) x_{a}+\left(k_{y}^{\prime}-k_{y}\right) y_{a}\right]\right) d k_{x}^{\prime} d k_{y}^{\prime} .
\end{aligned}
$$

The receiving spectrum $\mathbf{R}$ satisfies $\mathbf{R}\left(k_{x}, k_{y}, \omega\right) \cdot \mathbf{k}_{1}=0$, where $\mathbf{k}_{1}=k_{x} \hat{\mathbf{x}}+k_{y} \hat{\mathbf{y}}+\gamma_{1} \hat{\mathbf{z}}$, and the transmitting spectrum $\mathbf{T}$ satisfies $\mathbf{T}\left(k_{x}, k_{y}, \omega\right) \cdot \mathbf{k}_{1}^{-}=0$, where $\mathbf{k}_{1}^{-}=k_{x} \hat{\mathbf{x}}+k_{y} \hat{\mathbf{y}}-\gamma_{1} \hat{\mathbf{z}}$.

The effect of the multiple interactions between the antenna and the interface is included in the scattering matrix, comprising $\Gamma, \mathbf{T}, \mathbf{R}$, and $\overline{\mathbf{S}}$. These quantities depend on the electromagnetic properties of the lower medium, $\epsilon_{1}$ and $\sigma_{1}$, as well as the distance $z_{a}$ of the antenna from the interface. Therefore, the scattering matrix is a complicated nonlinear function of $\epsilon_{1}, \sigma_{1}$, and $z_{a}$. This explains why the distance $z_{a}$ of the antenna above the interface does not appear explicitly in (9) and (10), as was the case in (5) and (6) for free space. If the scattering matrix is determined for one set of $\epsilon_{1}, \sigma_{1}$, and $z_{a}$, it can only be used for this parameter set. If just one of the parameters is changed, one has to redetermine the scattering matrix. However, for many configurations the scattering matrix varies slowly with $\epsilon_{1}$ and $\sigma_{1}$ because the reflection coefficient describing the interface reflection varies slowly. This is illustrated numerically in Section VII.

If multiple interactions between objects in the lower medium and the AUT/interface system can be neglected, the scattering spectrum [the integral term in (10)] can be omitted from the formulation. Multiple interactions can be neglected if the objects are weakly scattering or if the lower medium is sufficiently lossy.

It is possible to determine the scattering matrix in (9) and (10) using the free-space scattering matrix in (5) and (6) as well as the reflection and transmission coefficients of the planar interface. However, since this procedure is complicated-because it involves solving an integral equation-and since the full free-space scattering matrix is difficult to determine (especially the scattering spectrum for evanescent waves), we will not recommend this procedure. Instead, we suggest the procedures presented in the following two sections.

\section{TRansmitting Spectrum in Terms OF A CURRENT DENSITY}

Assume that the downward-propagating field $\mathbf{E}_{1}^{-}(x, y, z, \omega)$ in the lower medium, radiated by the transmitting AUT when its position is given by $x_{a}=0, y_{a}=0$, and $z_{a} \geq 0$ and when 
it is excited with an incident field with voltage $V^{+}(\omega)$ in the reference plane, can be described in terms of the current density $\mathbf{J}(x, y, z, \omega)$. The relation between the electric field in the lower medium and the current density is [13, eq. (1)]

$$
\begin{array}{r}
\mathbf{E}_{1}^{-}(x, y, z, \omega)= \\
\cdot \mathbf{s} \mu_{0} \iiint_{z^{\prime} \geq 0} \overline{\mathbf{G}}\left(x-x^{\prime}, y-y^{\prime}, z, z^{\prime}, \omega\right) \\
\cdot \mathbf{J}\left(x^{\prime}, y^{\prime}, z^{\prime}, \omega\right) d x^{\prime} d y^{\prime} d z^{\prime}, \quad z<0 \quad(11)
\end{array}
$$

where the dyadic Green's function that accounts for the presence of the interface is as in (12), shown at the bottom of the page, and the dyadic $\overline{\mathbf{F}}$ is [13, (3)]

$$
\begin{aligned}
\overline{\mathbf{F}}\left(k_{x}, k_{y}, \omega\right)= & \frac{2}{\left(\gamma_{0}+\gamma_{1}\right)\left(k_{x}^{2}+k_{y}^{2}+\gamma_{0} \gamma_{1}\right)} \\
& \cdot\left[\hat{\mathbf{x}}\left(\left(k_{y}^{2}+\gamma_{0} \gamma_{1}\right) \hat{\mathbf{x}}-k_{x} k_{y} \hat{\mathbf{y}}+k_{x} \gamma_{1} \hat{\mathbf{z}}\right)\right. \\
& +\hat{\mathbf{y}}\left(-k_{x} k_{y} \hat{\mathbf{x}}+\left(k_{x}^{2}+\gamma_{0} \gamma_{1}\right) \hat{\mathbf{y}}+k_{y} \gamma_{1} \hat{\mathbf{z}}\right) \\
& \left.+\hat{\mathbf{z}}\left(k_{x} \gamma_{0} \hat{\mathbf{x}}+k_{y} \gamma_{0} \hat{\mathbf{y}}+\left(k_{x}^{2}+k_{y}^{2}\right) \hat{\mathbf{z}}\right)\right] .
\end{aligned}
$$

Inserting the expression for the Green's function (12) into the expression (11) for the electric field, one obtains

$$
\begin{array}{r}
\mathbf{E}_{1}^{-}(x, y, z, \omega)=\frac{-\omega \mu_{0}}{8 \pi^{2}} \iint_{-\infty}^{\infty} \overline{\mathbf{F}}\left(k_{x}, k_{y}, \omega\right) \tilde{\mathbf{J}}\left(k_{x}, k_{y},-\gamma_{0}, \omega\right) \\
\cdot \exp \left(i\left[k_{x} x+k_{y} y-\gamma_{1} z\right]\right) d k_{x} d k_{y} \quad \text { (14) }
\end{array}
$$

where $\tilde{\mathbf{J}}$ is the spatial Fourier transform of the current

$$
\begin{aligned}
\tilde{\mathbf{J}}\left(k_{x}, k_{y}, k_{z}, \omega\right)= & \iiint_{z \geq 0} \mathbf{J}(x, y, z, \omega) \\
& \cdot \exp \left(-i\left[k_{x} x+k_{y} y+k_{z} z\right]\right) d x d y d z .
\end{aligned}
$$

Comparing the expression (14) with the definition (10) of the transmitting spectrum with $x_{a}=y_{a}=0$, we find that

$$
\mathbf{T}\left(k_{x}, k_{y}, \omega\right)=\frac{-\omega \mu_{0}}{2 V^{+}(\omega)} \overline{\mathbf{F}}\left(k_{x}, k_{y}, \omega\right) \cdot \tilde{\mathbf{J}}\left(k_{x}, k_{y},-\gamma_{0}, \omega\right) .
$$

We will now explain how the current density $\mathbf{J}$ can be determined for different types of antennas. The Hertzian dipole has an impressed current density given by $\mathbf{J}(x, y, z, \omega)=\mathbf{p}(\omega) \delta(x) \delta(y) \delta\left(z-z_{a}\right)$ where $\delta$ denotes Dirac's delta function and $\mathbf{p}(\omega)$ is the dipole moment that depends on $V^{+}$. For more complicated antennas described by a nonimpressed current density, $\mathbf{J}$ can be determined from numerical methods, e.g., by solving the mixed-potential integral equation for a two-layer medium using the method of moments [7], [8] or by applying the finite-difference time-domain method [9]. In either case, the effect of multiple interactions between the antenna and the interface is included in the current density J. Moreover, semianalytical expressions exist for the current

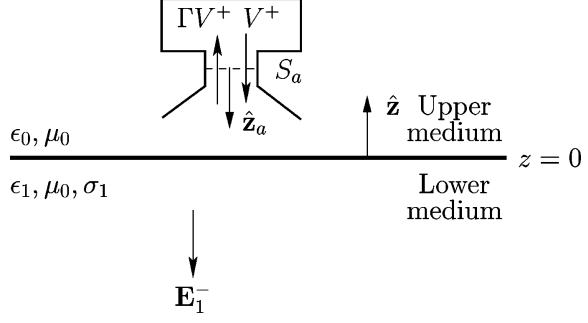

Fig. 4. Transmitting case considered to derive the reciprocity relation (30)

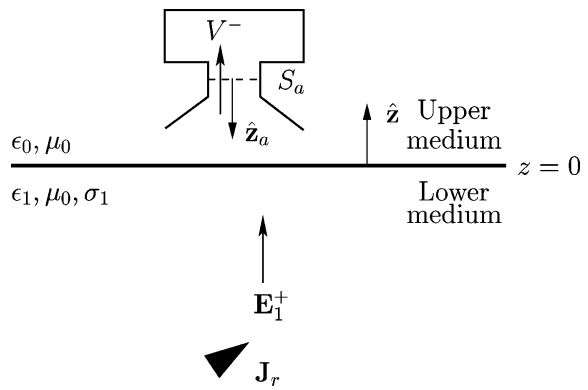

Fig. 5. Receiving case considered to derive the reciprocity relation (30).

density of some simple antennas, such as the horizontal wire antenna located a fraction of a wavelength from an interface [10, Sec. 1.9]. In Section VII we shall compute the transmitting spectrum for this wire antenna.

\section{Receiving SPectrum in Terms of THE TRANSMitTing SPECTRUM}

In this section, we use the reciprocity theorem to derive a relation between the transmitting and receiving spectra, $\mathbf{T}$ and $\mathbf{R}$ defined in (9) and (10), for a reciprocal AUT. The transmitting and receiving configurations are shown in Figs. 4 and 5. In the transmitting case, the voltage of the incident field in the reference plan is $V^{+}=V^{+}(\omega)$, and hence, the total voltage in the reference plane is $V=V(\omega)=V^{+}(1+\Gamma)$. The radiated electric field is denoted by $\mathbf{E}_{t}$. In the receiving case, it is assumed that a current density $\mathbf{J}_{r}$, producing the electric field $\mathbf{E}_{r}$, is located in the lower medium. It is furthermore assumed that the coaxial cable is attached to a matched receiver so that no incident field exists in the cable. The voltage of the emerging field in the reference plane is $V^{-}$. It is also assumed that no scattering objects are present in the lower medium.

Applying the reciprocity theorem [14, eq. (1.67)] to the region $z \geq 0$ yields

$$
\int_{S_{a}+S_{i}^{+}+S_{\infty}}\left(\mathbf{E}_{t} \times \mathbf{H}_{r}-\mathbf{E}_{r} \times \mathbf{H}_{t}\right) \cdot \hat{\mathbf{n}} d S=0 .
$$

Herein, $\hat{\mathbf{n}}$ is the outward normal unit vector, $S_{i}^{+}$is the surface just above the interface $z=0$, and $S_{\infty}$ is the half sphere $x^{2}+$ $y^{2}+z^{2}=r^{2}$ with $z \geq 0$ and infinite radius, $r \rightarrow \infty$. The

$\overline{\mathbf{G}}\left(x-x^{\prime}, y-y^{\prime}, z, z^{\prime}, \omega\right)=\frac{i}{8 \pi^{2}} \iint_{-\infty}^{\infty} \overline{\mathbf{F}}\left(k_{x}, k_{y}, \omega\right) \exp \left(i\left[k_{x}\left(x-x^{\prime}\right)+k_{y}\left(y-y^{\prime}\right)+\gamma_{0} z^{\prime}-\gamma_{1} z\right]\right) d k_{x} d k_{y}, \quad z<0, z^{\prime} \geq 0$ 
integral over $S_{\infty}$ can be shown to equal zero [14, p. 25]. The fields in the reference plane $S_{a}$ are

$$
\begin{aligned}
\mathbf{E}_{t} & =\frac{V^{+}(1+\Gamma) \hat{\boldsymbol{\rho}}_{a}}{\rho_{a} \ln \frac{b}{a}} \\
\mathbf{H}_{t} & =\frac{Y_{c} V^{+}(1-\Gamma) \hat{\boldsymbol{\phi}}_{a}}{2 \pi \rho_{a}} \\
\mathbf{E}_{r} & =\frac{V^{-} \hat{\boldsymbol{\rho}}_{a}}{\rho_{a} \ln \frac{b}{a}} \\
\mathbf{H}_{r} & =\frac{-Y_{c} V^{-} \hat{\boldsymbol{\phi}}_{a}}{2 \pi \rho_{a}} .
\end{aligned}
$$

Inserting these expressions into the reciprocity theorem (17) yields

$$
\begin{aligned}
\int_{S_{i}^{+}}\left(\mathbf{E}_{t} \times \mathbf{H}_{r}-\mathbf{E}_{r} \times \mathbf{H}_{t}\right) \cdot(-\hat{\mathbf{z}}) d S & =\int_{0}^{2 \pi} \int_{a}^{b} \\
\cdot \frac{-V^{+} V^{-} Y_{c}}{\pi \rho_{a}^{2} \ln \frac{b}{a}} \rho_{a} d \rho_{a} d \phi_{a} & =-2 Y_{c} V^{+} V^{-} .
\end{aligned}
$$

Inserting the expression (9) relating $V^{-}$to the receiving spectrum $\mathbf{R}$, and recalling that $V^{+}=0$ because the coaxial cable is connected to a matched receiver, we obtain

$$
\begin{array}{r}
\int_{S_{i}^{+}}\left(\mathbf{E}_{t} \times \mathbf{H}_{r}-\mathbf{E}_{r} \times \mathbf{H}_{t}\right) \cdot \hat{\mathbf{z}} d S=\frac{2 V^{+} Y_{c}}{(2 \pi)^{2}} \iint_{-\infty}^{\infty} \mathbf{R}\left(k_{x}, k_{y}, \omega\right) \\
\cdot \mathbf{S}_{1}^{+}\left(k_{x}, k_{y}, \omega\right) \exp \left(i\left[k_{x} x_{a}+k_{y} y_{a}\right]\right) d k_{x} d k_{y} .
\end{array}
$$

Here, $\mathbf{S}_{1}^{+}$is the plane-wave spectrum of the upward-propagating field $\mathbf{E}_{1}^{+}$in the lower medium. This upward-propagating field is given by [15, p. 386]

$$
\begin{aligned}
\mathbf{E}_{1}^{+}(x, y, z, \omega)= & \frac{-\omega \mu_{0}}{8 \pi^{2}} \iint_{-\infty}^{\infty} \frac{1}{\gamma_{1}}\left(\overline{\mathbf{I}}-\frac{\mathbf{k}_{1} \mathbf{k}_{1}}{k_{1}^{2}}\right) \cdot \int_{V_{1}} \mathbf{J}_{r}\left(x^{\prime}, y^{\prime}, z^{\prime}, \omega\right) \\
& \cdot \exp \left(i\left[k_{x}\left(x-x^{\prime}\right)+k_{y}\left(y-y^{\prime}\right)+\gamma_{1}\left(z-z^{\prime}\right)\right]\right) \\
& \cdot d x^{\prime} d y^{\prime} d z^{\prime} d k_{x} d k_{y}
\end{aligned}
$$

where $V_{1}$ denotes the region $z<0, \mathbf{k}_{1}=k_{x} \hat{\mathbf{x}}+k_{y} \hat{\mathbf{y}}+\gamma_{1} \hat{\mathbf{z}}$, and $z>z^{\prime}$. Comparing (24) with (8) reveals that the plane-wave spectrum $\mathbf{S}_{1}^{+}$is

$\mathbf{S}_{1}^{+}\left(k_{x}, k_{y}, \omega\right)=\frac{-\omega \mu_{0}}{2 \gamma_{1}}\left(\overline{\mathbf{I}}-\frac{\mathbf{k}_{1} \mathbf{k}_{1}}{k_{1}^{2}}\right) \cdot \int_{V_{1}} \mathbf{J}_{r}(x, y, z, \omega)$

- $\exp \left(-i\left[k_{x} x+k_{y} y+\gamma_{1} z\right]\right) d x d y d z$ and inserting into (23) gives (26), shown at the bottom of the page, where $\mathbf{R} \cdot \mathbf{k}_{1}=0$ has been used.

Applying the reciprocity theorem to the region $z<0$ yields

$$
\int_{S_{i}^{-}+S_{\infty}}\left(\mathbf{E}_{t} \times \mathbf{H}_{r}-\mathbf{E}_{r} \times \mathbf{H}_{t}\right) \cdot \hat{\mathbf{n}} d S=-\int_{V_{1}} \mathbf{E}_{t} \cdot \mathbf{J}_{r} d V
$$

where $S_{i}^{-}$denotes the surface just below the interface. Again, the integral over $S_{\infty}$ is zero. Using the expressions (7) and (10) with $\mathbf{S}_{1}^{+}=0$ for $\mathbf{E}_{t}$, and inserting into the reciprocity theorem (27), yields (28), shown at the bottom of the page. Using the boundary conditions for the electric and magnetic fields at the interface, it can readily be shown that

$\int_{S_{i}^{-}}\left(\mathbf{E}_{t} \times \mathbf{H}_{r}-\mathbf{E}_{r} \times \mathbf{H}_{t}\right) \cdot \hat{\mathbf{z}} d S=\int_{S_{i}^{+}}\left(\mathbf{E}_{t} \times \mathbf{H}_{r}-\mathbf{E}_{r} \times \mathbf{H}_{t}\right) \cdot \hat{\mathbf{z}} d S$.

Applying this result, and comparing (26) and (28), yields

$$
\mathbf{R}\left(k_{x}, k_{y}, \omega\right)=\frac{\gamma_{1}}{Y_{c} \omega \mu_{0}} \mathbf{T}\left(-k_{x},-k_{y}, \omega\right) .
$$

This final expression, relating the receiving spectrum to the transmitting spectrum, has the same form as the analogous free-space formula [2, eq. (6.55)]. The relation (30) between the transmitting and receiving spectra was derived for the case in which a coaxial cable is attached to the antenna. However, the relation is general and holds for any type of electromagnetic waveguide. For waveguides that do not support a TEM mode, the quantities $V^{+}$and $V^{-}$denote modal coefficients and not voltages [2, p. 255].

To derive (30), we have assumed that no scattering objects, including antennas, are present in the lower medium. This assumption is used in (27) when setting $\mathbf{E}_{t}$ in the lower medium equal to the background field (i.e., the field in the absence of scattering objects). If objects are present in the lower medium, the reciprocity relation (30) still holds provided these objects are weakly scattering, or the conductivity is sufficiently high to make the scattering from the objects negligible. In many applications, e.g., in GPR imaging [4], the field radiated by the transmitting AUT is assumed independent of objects in the lower medium. Hence, the downward-propagating field equals the background field, and consequently, the application of the reciprocity relation (30) is exact in these applications.

$$
\begin{aligned}
\int_{S_{i}^{+}}\left(\mathbf{E}_{t} \times \mathbf{H}_{r}-\mathbf{E}_{r} \times \mathbf{H}_{t}\right) \cdot \hat{\mathbf{z}} d S=\frac{-\omega \mu_{0} V^{+} Y_{c}}{(2 \pi)^{2}} \iint_{-\infty}^{\infty} \frac{1}{\gamma_{1}} \mathbf{R}\left(k_{x}, k_{y}, \omega\right) \cdot \int_{V_{1}} \mathbf{J}_{r}(x, y, z, \omega) \\
\cdot \exp \left(-i\left[k_{x} x+k_{y} y+\gamma_{1} z\right]\right) d x d y d z \exp \left(i\left[k_{x} x_{a}+k_{y} y_{a}\right]\right) d k_{x} d k_{y}
\end{aligned}
$$

$$
\begin{aligned}
\int_{S_{i}^{-}}\left(\mathbf{E}_{t} \times \mathbf{H}_{r}-\mathbf{E}_{r} \times \mathbf{H}_{t}\right) \cdot \hat{\mathbf{z}} d S=\frac{-V^{+}}{(2 \pi)^{2}} \iint_{-\infty}^{\infty} \mathbf{T}\left(-k_{x},-k_{y}, \omega\right) \cdot \int_{V_{1}} \mathbf{J}_{r}(x, y, z, \omega) \\
\cdot \exp \left(-i\left[k_{x} x+k_{y} y+\gamma_{1} z\right]\right) d x d y d z \exp \left(i\left[k_{x} x_{a}+k_{y} y_{a}\right]\right) d k_{x} d k_{y}
\end{aligned}
$$




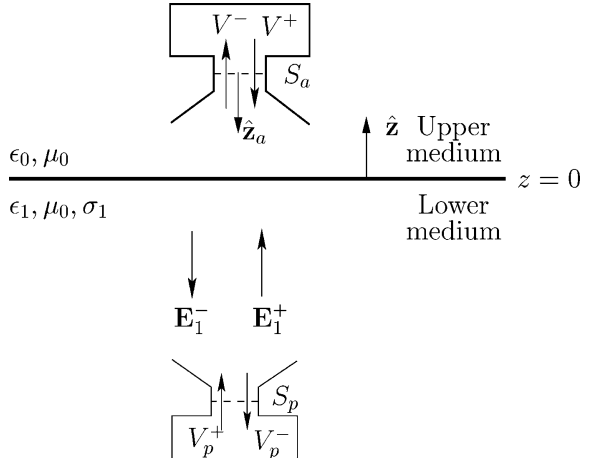

Fig. 6. Configuration used to determine the transmitting and receiving spectra by means of a probe located in the lower medium.

\section{Measuring the Plane-WaVe Spectra of the AUT}

In this section, we will show how the plane-wave transmitting and receiving spectra of Section III can be determined from measurements of the field in the lower medium using a small probe (this measurement procedure has been suggested in [11] for GPR applications). We assume that the small probe is located in the lower medium at the fixed position given by $x=x_{p}$, $y=y_{p}$, and $z=z_{p}$ with $z_{p}<0$ (see Fig. 6) and that it measures two orthogonal components of the electric field. The probe has the free-space transmitting spectrum $\mathbf{T}_{p}^{x}\left(k_{x}, k_{y}, \omega\right)$ (see Section II for the definition ${ }^{2}$ ) when a characteristic line of the probe is directed along $\hat{\mathbf{x}}$ and the free-space transmitting spectrum $\mathbf{T}_{p}^{y}\left(k_{x}, k_{y}, \omega\right)$ when the same characteristic line of the probe is directed along $\hat{\mathbf{y}}$. Also, the probe has the free-space receiving spectrum $\mathbf{R}_{p}^{x}\left(k_{x}, k_{y}, \omega\right)$ when a characteristic line of the probe is directed along $\hat{\mathbf{x}}$ and the free-space receiving spectrum $\mathbf{R}_{p}^{y}\left(k_{x}, k_{y}, \omega\right)$ when the same characteristic line of the probe is directed along $\hat{\mathbf{y}}$. The probe is attached to a coaxial cable with characteristic admittance $Y_{c p}$. For a reciprocal probe located in the lower medium, the transmitting and receiving spectra are related as

$$
\begin{aligned}
& \mathbf{R}_{p}^{x}\left(k_{x}, k_{y}, \omega\right)=\frac{\gamma_{1}}{Y_{c p} \omega \mu_{0}} \mathbf{T}_{p}^{x}\left(-k_{x},-k_{y}, \omega\right) \\
& \mathbf{R}_{p}^{y}\left(k_{x}, k_{y}, \omega\right)=\frac{\gamma_{1}}{Y_{c p} \omega \mu_{0}} \mathbf{T}_{p}^{y}\left(-k_{x},-k_{y}, \omega\right) .
\end{aligned}
$$

It is assumed that just one propagating TEM mode is excited in the cable. In addition, we assume that multiple interactions between the probe and the AUT/interface system can be neglected. Hence, the scattering spectra of the AUT and the probe can be omitted from the formulation. Neglecting these multiple interactions is usually a good approximation in practice when the probe is small or the lower medium is lossy.

\section{A. Computing the Transmitting Spectrum of the AUT}

The transmitting AUT is excited by an incident field in the coaxial cable with voltage $V^{+}$in the reference plane. The coaxial cable of the probe is attached to a matched receiver, so that no incident field exists in the cable. The emerging field with voltage $V_{p}^{x-}$ in the reference plane $S_{p}$ of the $\hat{\mathbf{x}}$-directed

\footnotetext{
${ }^{2}$ The free-space transmitting and receiving spectra for the probe are determined when the probe is located in a homogeneous medium with permittivity $\epsilon_{1}$ and conductivity $\sigma_{1}$
}

probe is a function of the transmitting antenna position, $x_{a}$ and $y_{a}$. Hence, by neglecting multiple interactions between the probe and the AUT/interface system, we get

$$
\begin{aligned}
V_{p}^{x-}\left(x_{a}, y_{a}, \omega\right)= & \frac{V^{+}}{(2 \pi)^{2}} \iint_{-\infty}^{\infty} \mathbf{R}_{p}^{x}\left(k_{x}, k_{y}, \omega\right) \cdot \mathbf{T}\left(k_{x}, k_{y}, \omega\right) \\
& \cdot \exp \left(i \left[k_{x}\left(x_{p}-x_{a}\right)+k_{y}\right.\right. \\
& \left.\left.\cdot\left(y_{p}-y_{a}\right)-\gamma_{1} z_{p}\right]\right) d k_{x} d k_{y} \cdot
\end{aligned}
$$

Similarly, the voltage $V_{p}^{y-}$ in the reference plane of the $\hat{\mathbf{y}}$-directed probe is

$$
\begin{aligned}
V_{p}^{y-}\left(x_{a}, y_{a}, \omega\right)= & \frac{V^{+}}{(2 \pi)^{2}} \iint_{-\infty}^{\infty} \mathbf{R}_{p}^{y}\left(k_{x}, k_{y}, \omega\right) \cdot \mathbf{T}\left(k_{x}, k_{y}, \omega\right) \\
& \cdot \exp \left(i \left[k_{x}\left(x_{p}-x_{a}\right)+k_{y}\right.\right. \\
& \left.\left.\cdot\left(y_{p}-y_{a}\right)-\gamma_{1} z_{p}\right]\right) d k_{x} d k_{y} .
\end{aligned}
$$

Fourier transforming $V_{p}^{x-}\left(x_{a}, y_{a}, \omega\right)$ with respect to $x_{a}$ and $y_{a}$, according to

$$
\begin{aligned}
\tilde{V}_{p}^{x-}\left(k_{x}, k_{y}, \omega\right) \iint_{-\infty}^{\infty} V_{p}^{x-}\left(x_{a}, y_{a}, \omega\right) \\
\cdot \exp \left(-i\left[k_{x} x_{a}+k_{y} y_{a}\right]\right) d x_{a} d y_{a}
\end{aligned}
$$

gives

$$
\begin{aligned}
& \tilde{V}_{p}^{x-}\left(k_{x}, k_{y}, \omega\right)=V^{+} \mathbf{R}_{p}^{x}\left(-k_{x},-k_{y}, \omega\right) \\
& \cdot \mathbf{T}\left(-k_{x},-k_{y}, \omega\right) \exp \left(-i\left[k_{x} x_{p}+k_{y} y_{p}+\gamma_{1} z_{p}\right]\right) .
\end{aligned}
$$

Similarly

$$
\begin{aligned}
& \tilde{V}_{p}^{y-}\left(k_{x}, k_{y}, \omega\right)=V^{+} \mathbf{R}_{p}^{y}\left(-k_{x},-k_{y}, \omega\right) \\
& \cdot \mathbf{T}\left(-k_{x},-k_{y}, \omega\right) \exp \left(-i\left[k_{x} x_{p}+k_{y} y_{p}+\gamma_{1} z_{p}\right]\right) .
\end{aligned}
$$

In the expression (35) for the Fourier transform, the integration extends over an entire plane perpendicular to the $z$ axis. In practice, however, the integration covers only a finite scan plane. In Section VII, we discuss the impact of the size of the scan plane on the accuracy of the measured spectra.

Using $\mathbf{T}\left(k_{x}, k_{y}, \omega\right)=T_{x} \hat{\mathbf{x}}+T_{y} \hat{\mathbf{y}}+T_{z} \hat{\mathbf{z}}, \mathbf{R}_{p}^{x}\left(k_{x}, k_{y}, \omega\right)=$ $R_{p x}^{x} \hat{\mathbf{x}}+R_{p y}^{x} \hat{\mathbf{y}}+R_{p z}^{x} \hat{\mathbf{z}}$, and similarly for $\mathbf{R}_{p}^{y}$, along with $\mathbf{k}_{1}^{-} \cdot \mathbf{T}=$ $0, \mathbf{k}_{1}^{-} \cdot \mathbf{R}_{p}^{x}=0$, and $\mathbf{k}_{1}^{-} \cdot \mathbf{R}_{p}^{y}=0$ with $\mathbf{k}_{1}^{-}=k_{x} \hat{\mathbf{x}}+k_{y} \hat{\mathbf{y}}-\gamma_{1} \hat{\mathbf{z}}$, we can determine $T_{x}$ and $T_{y}$ from (36) and (37)

$$
\begin{aligned}
T_{x} & =\frac{\tilde{V}_{p}^{x-}\left(-k_{x},-k_{y}, \omega\right) C_{t 22}-\tilde{V}_{p}^{y-}\left(-k_{x},-k_{y}, \omega\right) C_{t 12}}{D_{t}} \\
T_{y} & =\frac{\tilde{V}_{p}^{y-}\left(-k_{x},-k_{y}, \omega\right) C_{t 11}-\tilde{V}_{p}^{x-}\left(-k_{x},-k_{y}, \omega\right) C_{t 21}}{D_{t}}
\end{aligned}
$$

where

$$
\begin{aligned}
C_{t 11} & =C_{t}\left(R_{p x}^{x}\left(\gamma_{1}^{2}+k_{x}^{2}\right)+R_{p y}^{x} k_{x} k_{y}\right) \\
C_{t 12} & =C_{t}\left(R_{p y}^{x}\left(\gamma_{1}^{2}+k_{y}^{2}\right)+R_{p x}^{x} k_{x} k_{y}\right) \\
C_{t 21} & =C_{t}\left(R_{p x}^{y}\left(\gamma_{1}^{2}+k_{x}^{2}\right)+R_{p y}^{y} k_{x} k_{y}\right) \\
C_{t 22} & =C_{t}\left(R_{p y}^{y}\left(\gamma_{1}^{2}+k_{y}^{2}\right)+R_{p x}^{y} k_{x} k_{y}\right) \\
C_{t} & =V^{+} \exp \left(i\left[k_{x} x_{p}+k_{y} y_{p}-\gamma_{1} z_{p}\right]\right) \\
D_{t} & =k_{1}^{2} C_{t}^{2}\left(R_{p x}^{x} R_{p y}^{y}-R_{p x}^{y} R_{p y}^{x}\right) .
\end{aligned}
$$




\section{B. Computing the Receiving Spectrum of the AUT}

The probe is excited by an incident field in the coaxial cable with voltage $V_{p}^{+}$in the reference plane $S_{p}$. When the probe is $\hat{\mathbf{x}}$ directed, an emerging field with voltage $V^{x-}$ exists in the reference plane $S_{a}$ of the AUT. Similarly, when the probe is $\hat{\mathbf{y}}$ directed, an emerging field with voltage $V^{y-}$ exists in the reference plane. The AUT is connected to a matched receiver, so that no incident field exists in the coaxial cable. When neglecting multiple interactions between the probe and the AUT/interface system, the above-defined voltages are

$$
\begin{aligned}
& V^{x-}\left(x_{a}, y_{a}, \omega\right)= \frac{V_{p}^{+}}{(2 \pi)^{2}} \iint_{-\infty}^{\infty} \mathbf{R}\left(k_{x}, k_{y}, \omega\right) \cdot \mathbf{T}_{p}^{x}\left(k_{x}, k_{y}, \omega\right) \\
& \cdot \exp \left(i \left[k_{x}\left(x_{a}-x_{p}\right)+k_{y}\right.\right. \\
&\left.\left.\cdot\left(y_{a}-y_{p}\right)-\gamma_{1} z_{p}\right]\right) d k_{x} d k_{y} \\
& V^{y-}\left(x_{a}, y_{a}, \omega\right)= \frac{V_{p}^{+}}{(2 \pi)^{2}} \iint_{-\infty}^{\infty} \mathbf{R}\left(k_{x}, k_{y}, \omega\right) \cdot \mathbf{T}_{p}^{y}\left(k_{x}, k_{y}, \omega\right) \\
& \cdot \exp \left(i \left[k_{x}\left(x_{a}-x_{p}\right)+k_{y}\right.\right. \\
&\left.\left.\cdot\left(y_{a}-y_{p}\right)-\gamma_{1} z_{p}\right]\right) d k_{x} d k_{y} \cdot
\end{aligned}
$$

Fourier transforming with respect to $x_{a}$ and $y_{a}$ yields

$$
\begin{array}{r}
\tilde{V}^{x-}\left(k_{x}, k_{y}, \omega\right)=V_{p}^{+} \mathbf{R}\left(k_{x}, k_{y}, \omega\right) \cdot \mathbf{T}_{p}^{x}\left(k_{x}, k_{y}, \omega\right) \\
\cdot \exp \left(-i\left[k_{x} x_{p}+k_{y} y_{p}+\gamma_{1} z_{p}\right]\right) \\
\tilde{V}^{y-}\left(k_{x}, k_{y}, \omega\right)= \\
V_{p}^{+} \mathbf{R}\left(k_{x}, k_{y}, \omega\right) \cdot \mathbf{T}_{p}^{y}\left(k_{x}, k_{y}, \omega\right) \\
\cdot \exp \left(-i\left[k_{x} x_{p}+k_{y} y_{p}+\gamma_{1} z_{p}\right]\right) .
\end{array}
$$

Using $\mathbf{R}\left(k_{x}, k_{y}, \omega\right)=R_{x} \hat{\mathbf{x}}+R_{y} \hat{\mathbf{y}}+R_{z} \hat{\mathbf{z}}, \mathbf{T}_{p}^{x}\left(k_{x}, k_{y}, \omega\right)=$ $T_{p x}^{x} \hat{\mathbf{x}}+T_{p y}^{x} \hat{\mathbf{y}}+T_{p z}^{x} \hat{\mathbf{z}}$, and similarly for $\mathbf{T}_{p}^{y}$, along with $\mathbf{k}_{1} \cdot \mathbf{R}=0$, $\mathbf{k}_{1} \cdot \mathbf{T}_{p}^{x}=0$, and $\mathbf{k}_{1} \cdot \mathbf{T}_{p}^{y}=0$ with $\mathbf{k}_{1}=k_{x} \hat{\mathbf{x}}+k_{y} \hat{\mathbf{y}}+\gamma_{1} \hat{\mathbf{z}}$, we can determine $R_{x}$ and $R_{y}$ from (48) and (49)

$$
\begin{aligned}
R_{x} & =\frac{\tilde{V}^{x-}\left(k_{x}, k_{y}, \omega\right) C_{r 22}-\tilde{V}^{y-}\left(k_{x}, k_{y}, \omega\right) C_{r 12}}{D_{r}} \\
R_{y} & =\frac{\tilde{V}^{y-}\left(k_{x}, k_{y}, \omega\right) C_{r 11}-\tilde{V}^{x-}\left(k_{x}, k_{y}, \omega\right) C_{r 21}}{D_{r}}
\end{aligned}
$$

where

$$
\begin{aligned}
C_{r 11} & =C_{r}\left(T_{p x}^{x}\left(\gamma_{1}^{2}+k_{x}^{2}\right)+T_{p y}^{x} k_{x} k_{y}\right) \\
C_{r 12} & =C_{r}\left(T_{p y}^{x}\left(\gamma_{1}^{2}+k_{y}^{2}\right)+T_{p x}^{x} k_{x} k_{y}\right) \\
C_{r 21} & =C_{r}\left(T_{p x}^{y}\left(\gamma_{1}^{2}+k_{x}^{2}\right)+T_{p y}^{y} k_{x} k_{y}\right) \\
C_{r 22} & =C_{r}\left(T_{p y}^{y}\left(\gamma_{1}^{2}+k_{y}^{2}\right)+T_{p x}^{y} k_{x} k_{y}\right) \\
C_{r} & =V_{p}^{+} \exp \left(-i\left[k_{x} x_{p}+k_{y} y_{p}+\gamma_{1} z_{p}\right]\right) \\
D_{r} & =k_{1}^{2} C_{r}^{2}\left(T_{p x}^{x} T_{p y}^{y}-T_{p x}^{y} T_{p y}^{x}\right) .
\end{aligned}
$$

To verify the expressions for the transmitting and receiving spectra, we assume that both the AUT and the probe are reciprocal. Insert the expressions (50) and (51) for $R_{x}$ and $R_{y}$, as well as (38) and (39) for $T_{x}$ and $T_{y}$ into the reciprocity relation (30), and use the reciprocity relations (31) and (32) for the probe in order to get

$$
\frac{V^{x-} Y_{c}}{V_{p}^{+}}=\frac{V_{p}^{x-} Y_{c p}}{V^{+}} \quad \frac{V^{y-} Y_{c}}{V_{p}^{+}}=\frac{V_{p}^{y-} Y_{c p}}{V^{+}} .
$$

These relations state that the ratio between the current of the emerging field in the reference plane of the antenna and the voltage of the incident field in the reference plane of the probe equals the ratio between the current of the emerging field in the reference plane of the probe and the voltage of the incident field in the reference plane of the antenna. The relations (58) can also be derived directly using the reciprocity theorem following a procedure similar to the one outlined in Section V [2, p. 266], [14, Sec. 4.2]. Remarkably, (58) holds even if strong scatterers are present in the lower medium.

\section{NUMERICAL SIMULATIONS}

In this section, we illustrate some of the concepts of the paper through numerical simulations. We consider an AUT consisting of a center-fed horizontal $\hat{\mathbf{x}}$-directed wire with length $l=0.48 \lambda_{0}\left(\lambda_{0}=2 \pi / k_{0}\right.$ being the wavelength in the upper medium), wire radius $a=3 \cdot 10^{-3} \lambda_{0}$, and feed point at the height $z_{a}$ above the interface. The coaxial cable attached to the antenna has the characteristic admittance $Y_{c}=20 \mathrm{mS}$. For this configuration, a semianalytical expression for the current density $\mathbf{J}$ on the antenna exists [10, Sec. 1.9]. Placing the reference plane $S_{a}$ at the feed point, and using the expression (16) for the plane-wave transmitting spectrum, yields

$$
\begin{gathered}
\mathbf{T}\left(k_{x}, k_{y}, \omega\right)=\frac{i \omega \mu_{0}(1+\Gamma) k_{L} \exp \left(i \gamma_{0} z_{a}\right)\left(\cos \frac{k_{x} l}{2}-\cos \frac{k_{L} l}{2}\right)}{Z \cos \frac{k_{L} l}{2}\left(k_{L}^{2}-k_{x}^{2}\right)\left(\gamma_{0}+\gamma_{1}\right)\left(k_{x}^{2}+k_{y}^{2}+\gamma_{0} \gamma_{1}\right)} \\
\cdot\left[\hat{\mathbf{x}}\left(k_{y}^{2}+\gamma_{0} \gamma_{1}\right)-\hat{\mathbf{y}} k_{x} k_{y}+\hat{\mathbf{z}} k_{x} \gamma_{0}\right]
\end{gathered}
$$

where $\gamma_{0}$ and $\gamma_{1}$ are defined previously and

$$
\begin{aligned}
& k_{L}=k_{L}(\omega) \\
&=k_{0}\left\{1+\frac{2}{\ln \left(\frac{2 z_{a}}{a}\right)}\left[\frac{1}{\left(2 k_{1} z_{a}\right)^{2}}-\frac{K_{1}\left(2 k_{1} z_{a}\right)}{2 k_{1} z_{a}}+i \pi \frac{I_{1}\left(2 k_{1} z_{a}\right)}{4 k_{1} z_{a}}\right.\right. \\
&-i\left(\frac{2 k_{1} z_{a}}{3}+\frac{\left(2 k_{1} z_{a}\right)^{3}}{45}\right. \\
&\left.\left.\left.+\frac{\left(2 k_{1} z_{a}\right)^{5}}{1575}+\cdots\right)\right]\right\}^{1 / 2}
\end{aligned}
$$

with $I_{1}$ and $K_{1}$ being the modified Bessel functions of the first order and the first and second kind, respectively. Moreover,

$$
Z=\frac{\omega \mu_{0} k_{L}}{2 \pi k_{0}^{2}} \ln \frac{2 z_{a}}{a}
$$

and the reflection coefficient $\Gamma$ is

$$
\Gamma=\Gamma(\omega)=\frac{Y_{c}-Y_{a}(\omega)}{Y_{c}+Y_{a}(\omega)}
$$

with the antenna admittance

$$
Y_{a}(\omega)=\frac{-i}{2 Z} \tan \frac{k_{L} l}{2} .
$$

The above expressions are valid when the antenna height $z_{a}$ is less than $\lambda_{0} / 4$. As expected, the plane-wave transmitting spectrum in (59) depends in a nontrivial manner on the antenna height and the electromagnetic properties of the lower medium. 


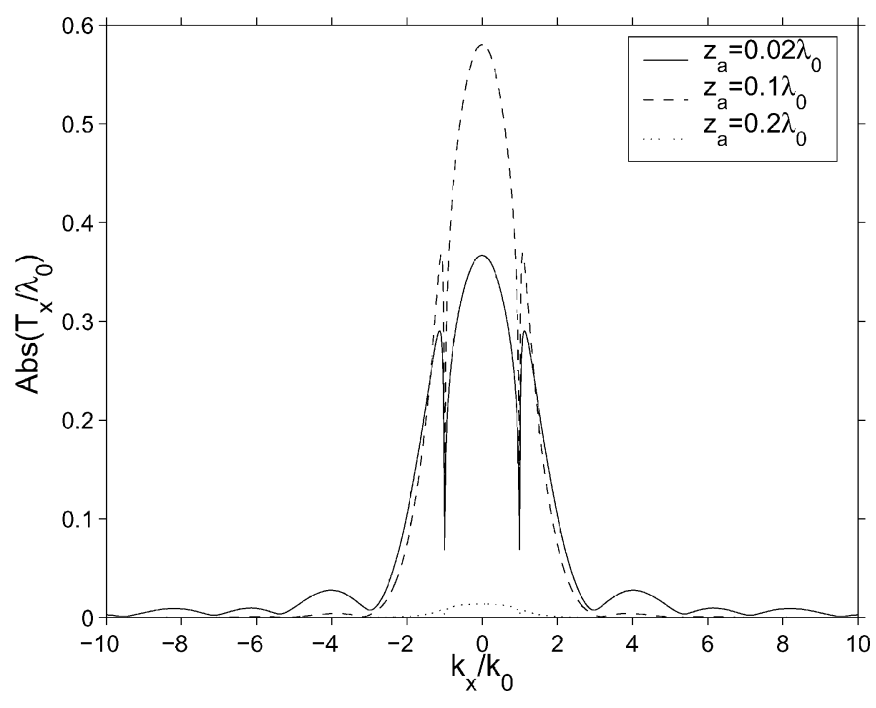

Fig. 7. Amplitude of $T_{x} / \lambda_{0}$ for $k_{y}=0, k_{1}^{2}=(8+3 i) k_{0}^{2}$, and three different heights $z_{a}$ of the AUT above the interface.

The factor $\exp \left(i \gamma_{0} z_{a}\right)$ in (59) causes the plane-wave spectrum $\mathbf{T}$ to be an exponentially decaying function of $k_{x}^{2}+k_{y}^{2}$ in the spectral region $k_{x}^{2}+k_{y}^{2}>k_{0}^{2}$. This spectral region corresponds to evanescent waves in the air. Hence, the spectral bandwidth of the plane-wave spectrum depends on the distance $z_{a}$ between the antenna and the interface. Fig. 7 shows the amplitude of the $\hat{\mathbf{x}}$-component $T_{x} / \lambda_{0}$ of the frequency-normalized transmitting spectrum as a function of $k_{x} / k_{0}$ for $k_{y}=0$ (note that the $\hat{\mathbf{y}}$-component of the transmitting spectrum is zero for $\left.k_{y}=0\right)$. In this plot, $k_{1}^{2}=(8+3 i) k_{0}^{2}$ corresponding to $\epsilon_{1}=8 \epsilon_{0}$ and $\sigma_{1} /\left(\epsilon_{0} \omega\right)=3$ (e.g., $\sigma_{1}=0.05 \mathrm{~S} / \mathrm{m}$ at $300 \mathrm{MHz}$ ). It is observed that the spectral bandwidth decreases as $z_{a}$ increases. Also, it is seen that the transmitting spectrum has a local minimum for $\left|k_{x} / k_{0}\right|=1$, corresponding to a plane-wave propagation direction in the lower medium determined by the critical angle $\theta_{c}=\arcsin \left(\sqrt{\epsilon_{0} / \epsilon_{1}}\right)$ measured from the negative $z$ axis. This agrees with the fact that the E-plane far-field pattern in the direction given by the critical angle has a minimum [3, Fig. 4.3] because the far-field pattern in this direction is determined from the plane-wave transmitting spectrum for $\left|k_{x}\right|=k_{0}$ and $k_{y}=0$ [16, pp. 284-286].

We will now investigate how the transmitting spectrum depends on the electromagnetic properties of the lower medium. To this end, we assume that the AUT is at the height $z_{a}=$ $0.02 \lambda_{0}$ above the interface and that $\sigma_{1} /\left(\epsilon_{0} \omega\right)=3$. Figs. 8 and 9 show the amplitude and phase of $T_{x} / \lambda_{0}$, respectively, when the permittivity $\epsilon_{1}$ of the lower medium equals $6 \epsilon_{0}, 8 \epsilon_{0}$, and $10 \epsilon_{0}$. For this parameter range, $T_{x} / \lambda_{0}$ changes only little with respect to $\epsilon_{1}$. Moreover, the amplitude of $T_{x} / \lambda_{0}$ changes less than the phase. By comparing with Fig. 7, we see that $\left|T_{x} / \lambda_{0}\right|$ is much more sensitive to variation in antenna height $z_{a}$ than to variation in permittivity $\epsilon_{1}$. We also found that $T_{x} / \lambda_{0}$ exhibit similar slow variation when $\epsilon_{1}=8 \epsilon_{0}$ and $\sigma_{1} /\left(\epsilon_{0} \omega\right)$ is varied from 0-6. For the sake of brevity, the plots resulting from this investigation are not shown here.

Next, we consider a simulation of a measurement of the plane-wave transmitting spectrum $\mathbf{T}$ for the case in which the AUT is $z_{a}=0.02 \lambda_{0}$ above the interface. The wavenumber $k_{1}$

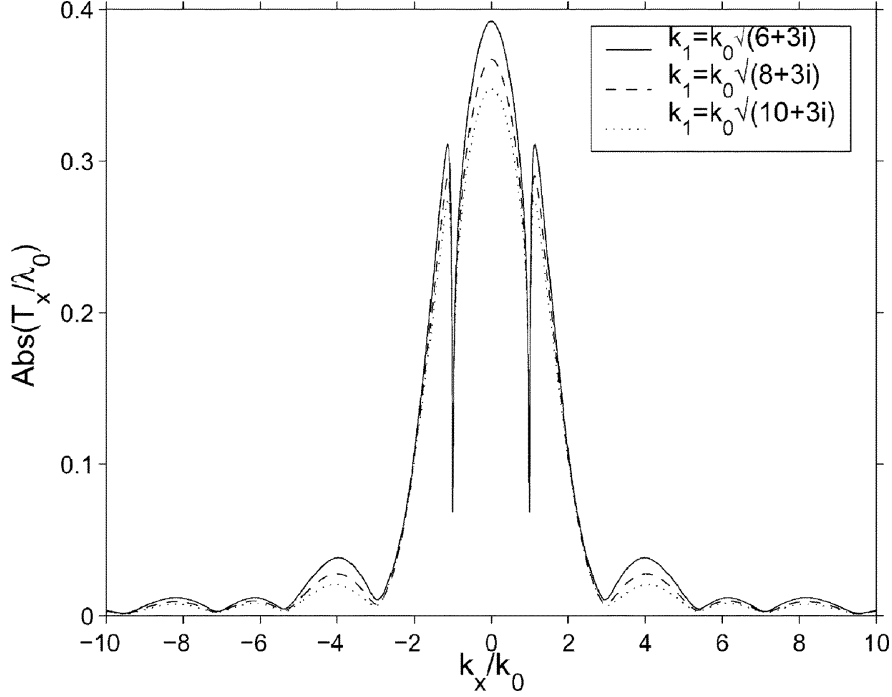

Fig. 8. Amplitude of $T_{x} / \lambda_{0}$ for $k_{y}=0, z_{a}=0.02 \lambda_{0}$, and three values of $k_{1}$

of the lower medium is given by $k_{1}^{2}=(8+3 i) k_{0}^{2}$, corresponding to $\epsilon_{1}=8 \epsilon_{0}$ and $\sigma_{1} /\left(\epsilon_{0} \omega\right)=3$. The voltage of the incident field in the reference plane of the AUT is $V^{+}=1 \mathrm{~V}$. The probe is a Hertzian dipole. Placing the reference plane at the feed point, the probe current density becomes $\mathbf{J}_{p}^{x}=-2 Y_{c p} V_{p}^{+} \hat{\mathbf{x}} \delta(x) \delta(y) \delta(z)$ and $\mathbf{J}_{p}^{y}=-2 Y_{c p} V_{p}^{+} \hat{\mathbf{y}} \delta(x) \delta(y) \delta(z)$ for the $\hat{\mathbf{x}}$ - and $\hat{\mathbf{y}}$-directed probe, respectively. Hence, using (31) and (32), the free-space plane-wave receiving spectra for the probe in the lower medium are

$$
\begin{aligned}
& \mathbf{R}_{p}^{x}\left(k_{x}, k_{y}, \omega\right)=\left[\hat{\mathbf{x}}-\frac{k_{x}}{k_{1}^{2}}\left(\hat{\mathbf{x}} k_{x}+\hat{\mathbf{y}} k_{y}-\hat{\mathbf{z}} \gamma_{1}\right)\right] \\
& \mathbf{R}_{p}^{y}\left(k_{x}, k_{y}, \omega\right)=\left[\hat{\mathbf{y}}-\frac{k_{y}}{k_{1}^{2}}\left(\hat{\mathbf{x}} k_{x}+\hat{\mathbf{y}} k_{y}-\hat{\mathbf{z}} \gamma_{1}\right)\right] .
\end{aligned}
$$

Note that $\mathbf{R}_{p}^{x} \cdot \mathbf{T}=T_{x}$ and $\mathbf{R}_{p}^{y} \cdot \mathbf{T}=T_{y}$. Hence, the voltage associated with the emerging field in the reference plane of the probe is proportional to the component of the electric field parallel to the dipole moment at the point of the dipole. This illustrates that the Hertzian dipole is an ideal probe.

Fig. 10 shows the frequency-normalized spatial Fourier transform $\tilde{V}_{p}^{x-} / \lambda_{1}\left(\lambda_{1}=2 \pi / \sqrt{\operatorname{Re}\left(k_{1}^{2}\right)}\right.$ is the wavelength in the lower medium) for $k_{y}=0$ of the voltage $V_{p}^{x-}$ of the emerging field in the reference plane of the probe, determined from (36). The Fourier transform is generally zero for $k_{x}^{2}+k_{y}^{2}>k_{\max }^{2}$ with $2 k_{\max }$ denoting the spectral bandwidth. As seen from Fig. 10 the spectral bandwidth decreases as $\left|z_{p}\right|$ increases. This can be understood from the fact that $\tilde{V}_{p}^{x-}$ contains the factor $\exp \left(-i \gamma_{1} z_{p}\right)$. For $k_{x}^{2}+k_{y}^{2}>\operatorname{Re}\left(k_{1}^{2}\right)$ this factor is exponentially decaying for increasing $k_{x}^{2}+k_{y}^{2}$, corresponding to evanescent waves in the lower medium. ${ }^{3}$ As a function of $k_{x}^{2}+k_{y}^{2}$ the decay increases as $\left|z_{p}\right|$ increases. The sampling distance in the scan plane is $\Delta_{x y}=\pi / k_{\max }$. If the probe is placed at a depth greater than $\lambda_{1}$, it can be assumed that $k_{\max }=\sqrt{\operatorname{Re}\left(k_{1}^{2}\right)}$, so that $\Delta_{x y}=\pi / \sqrt{\operatorname{Re}\left(k_{1}^{2}\right)}=\lambda_{1} / 2$.

\footnotetext{
${ }^{3}$ When the lower medium is lossy the factor $\exp \left(-i \gamma_{1} z_{p}\right)$ is exponentially decaying for increasing $k_{x}^{2}+k_{y}^{2}$ for all values of $k_{x}$ and $k_{y}$. However, the decay increases when $k_{x}^{2}+k_{y}^{2}>\operatorname{Re}\left(k_{1}^{2}\right)$.
} 


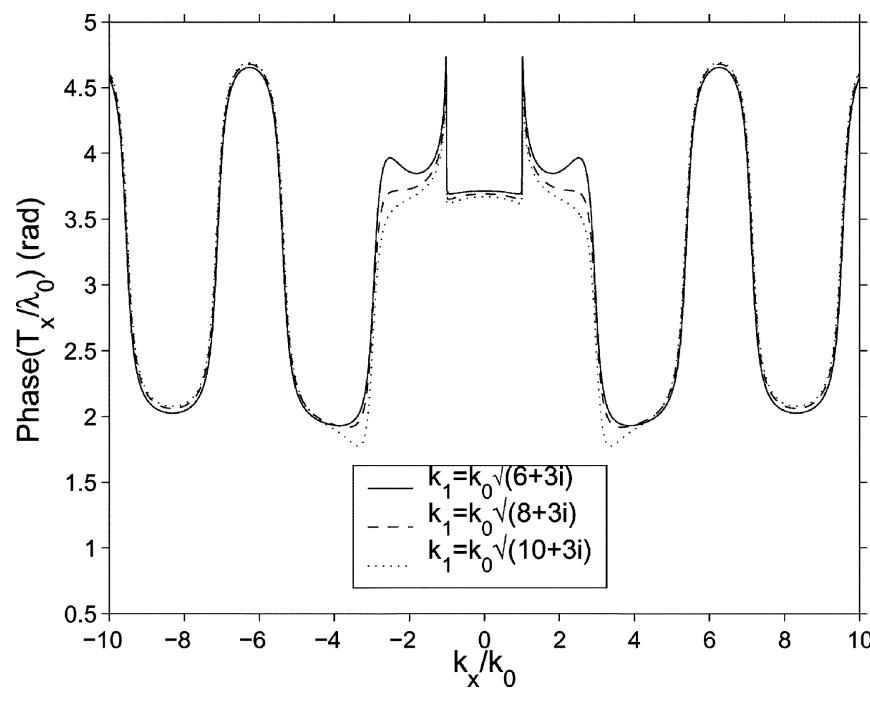

Fig. 9. Phase of $T_{x} / \lambda_{0}$ for $k_{y}=0, z_{a}=0.02 \lambda_{0}$, and three values of $k_{1}$.

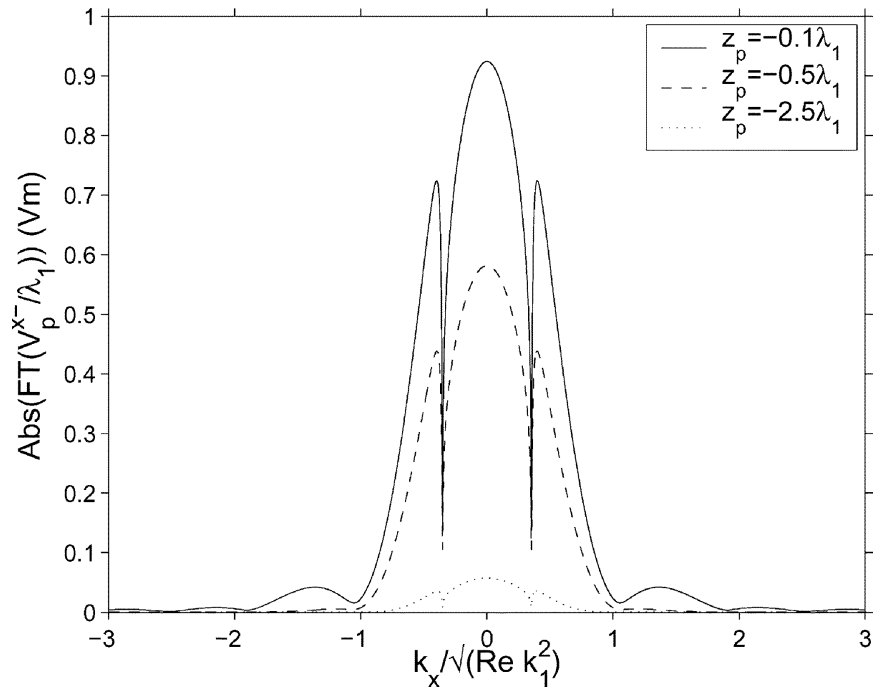

Fig. 10. Amplitude of the Fourier transform $\mathrm{FT}\left(V_{p}^{x-} / \lambda_{1}\right)=\tilde{V}_{p}^{x-} / \lambda_{1}$ for $k_{y}=0, z_{a}=0.02 \lambda_{0}$, and $k_{1}^{2}=(8+3 i) k_{0}^{2}$. The probe is positioned at $z=z_{p}$ where $z_{p}$ takes on the values $-0.1 \lambda_{1},-0.5 \lambda_{1}$, and $-2.5 \lambda_{1}$.

Fig. 11 shows the frequency-normalized amplitude $\left|\lambda_{1} V_{p}^{x-}\right|$ of the voltage of the emerging field in the reference plane of the probe, determined from (33), for $y_{a}=0$ and three values of the probe depth $z_{p}$. From Fig. 11, it is tempting to conclude that a scan plane size of $4 \lambda_{1}$ by $4 \lambda_{1}$ is sufficient to obtain an accurate estimate of the plane-wave spectrum. However, as will now be demonstrated, such a conclusion is erroneous because the probe outputs $V_{p}^{x-}$ and $V_{p}^{y-}$ oscillate rapidly and decrease slowly with increasing $x_{a}, y_{a}$. Figs. 12 and 13 show the amplitude and phase of $T_{x} / \lambda_{0}$, determined from (38), when the probe depth is $z_{p}=-\lambda_{1}$. The square scan plane is centered above the probe and its side length is $3 \lambda_{0}, 6 \lambda_{0}$, and $24 \lambda_{0}$, respectively. To minimize the truncation effect caused by effectively setting the probe outputs $V_{p}^{x-}$ and $V_{p}^{y-}$ equal to zero outside the scan plane, a Blackman window function is multiplied onto $V_{p}^{x-}$ and $V_{p}^{y-}$ before the Fourier transform is applied. It is observed that neither the amplitude nor the phase of $T_{x}$ can be determined accurately for large values of $\left|k_{x} / k_{0}\right|$. The value

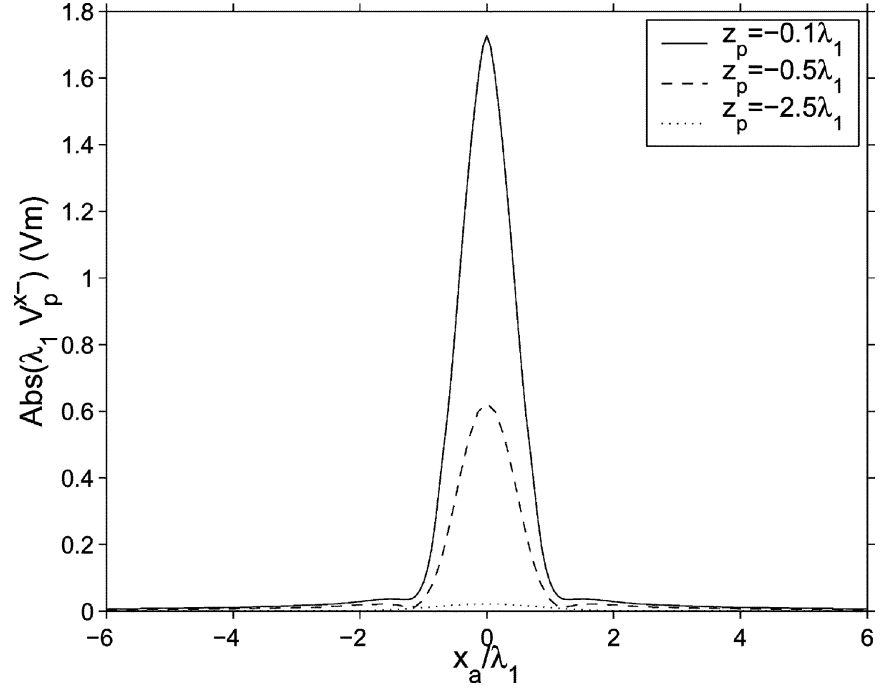

Fig. 11. Amplitude of $\lambda_{1} V_{p}^{x-}$ for $y_{a}=0, z_{a}=0.02 \lambda_{0}$, and $k_{1}^{2}=(8+$ $3 i) k_{0}^{2}$. The probe is positioned at $z=z_{p}$ where $z_{p}$ takes on the values $-0.1 \lambda_{1}$, $-0.5 \lambda_{1}$, and $-2.5 \lambda_{1}$.

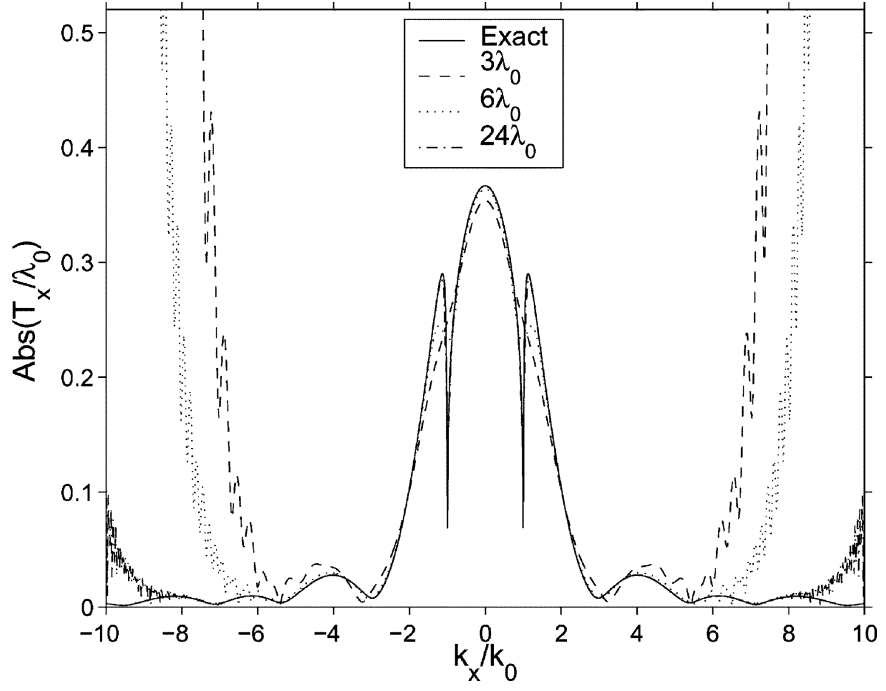

Fig. 12. Amplitude of the measured $T_{x} / \lambda_{0}$ for $k_{y}=0, z_{a}=0.02 \lambda_{0}, k_{1}^{2}=$ $(8+3 i) k_{0}^{2}$, and $z_{p}=-\lambda_{1}$. The side length of the square scan plane takes on the values $3 \lambda_{0}, 6 \lambda_{0}$, and $24 \lambda_{0}$.

of $\left|k_{x} / k_{0}\right|$, for which the measured $T_{x}$ deviates from the exact result, increases as the scan plane size increases. The reason for the deviation is that the expression for $T_{x}$ in (38) is proportional to $\exp \left(i \gamma_{1} z_{p}\right)$. This factor is exponentially increasing for $k_{x}^{2}+k_{y}^{2}>\operatorname{Re}\left(k_{1}^{2}\right)$, corresponding to evanescent waves in the lower medium. Consequently, the estimation of $T_{x}$ for evanescent waves in the lower medium is highly sensitive to noise and inaccuracies caused by a finite scan plane. In Figs. 14 and 15, the probe depth is $z_{p}=-1.5 \lambda_{1}$. Due to the larger probe depth, the evanescent waves are attenuated more than when $z_{p}=-\lambda_{1}$ and consequently, the value of $\left|k_{x} / k_{0}\right|$, for which the measured $T_{x}$ deviates from the exact value, is decreased.

Unfortunately, it is not possible to derive a general expression for the scan plane size required to compute the spectrum of the AUT to a certain accuracy. The scan plane size depends on the height $z_{a}$ of the AUT above the interface, the probe depth $z_{p}$, 


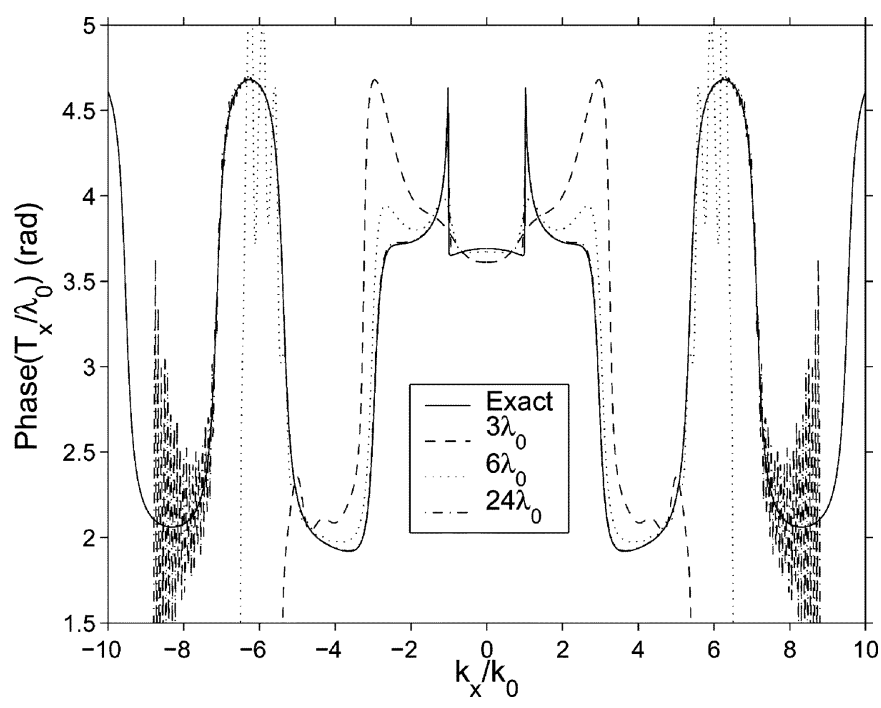

Fig. 13. Phase of the measured $T_{x} / \lambda_{0}$ for $k_{y}=0, z_{a}=0.02 \lambda_{0}, k_{1}^{2}=$ $(8+3 i) k_{0}^{2}$, and $z_{p}=-\lambda_{1}$. The side length of the square scan plane takes on the values $3 \lambda_{0}, 6 \lambda_{0}$, and $24 \lambda_{0}$.

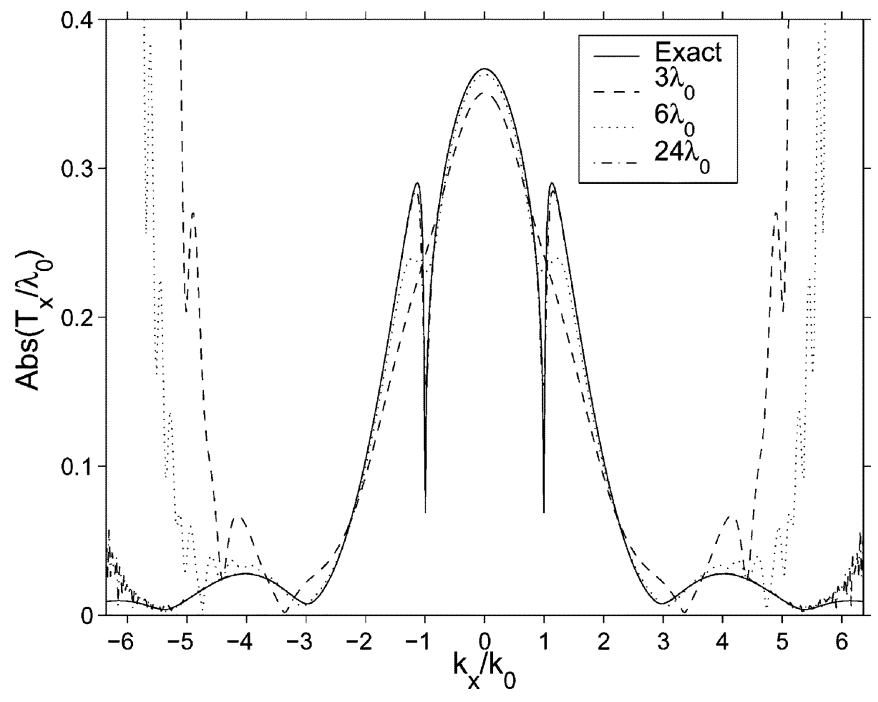

Fig. 14. Amplitude of the measured $T_{x} / \lambda_{0}$ for $k_{y}=0, z_{a}=0.02 \lambda_{0}, k_{1}^{2}=$ $(8+3 i) k_{0}^{2}$, and $z_{p}=-1.5 \lambda_{1}$. The side length of the square scan plane takes on the values $3 \lambda_{0}, 6 \lambda_{0}$, and $24 \lambda_{0}$.

the electromagnetic properties of the lower medium, and the radiation properties of the AUT.

\section{SUMMARY AND FUTURE WORK}

An antenna close to a planar interface (the AUT) was characterized by a plane-wave scattering matrix for the "total" system that includes both the antenna and the interface. With this formulation, the multiple interactions between the antenna and the interface are implicitly included in the scattering matrix of the antenna/interface system (multiple interactions are difficult to describe with the standard free-space scattering matrix formulation). It was shown how the plane-wave transmitting spectrum can be determined from the current density of the antenna. For ideal antennas such as the Hertzian dipole, the current density is impressed and not affected by the interface. For realistic antennas, the current density can be determined with numerical or

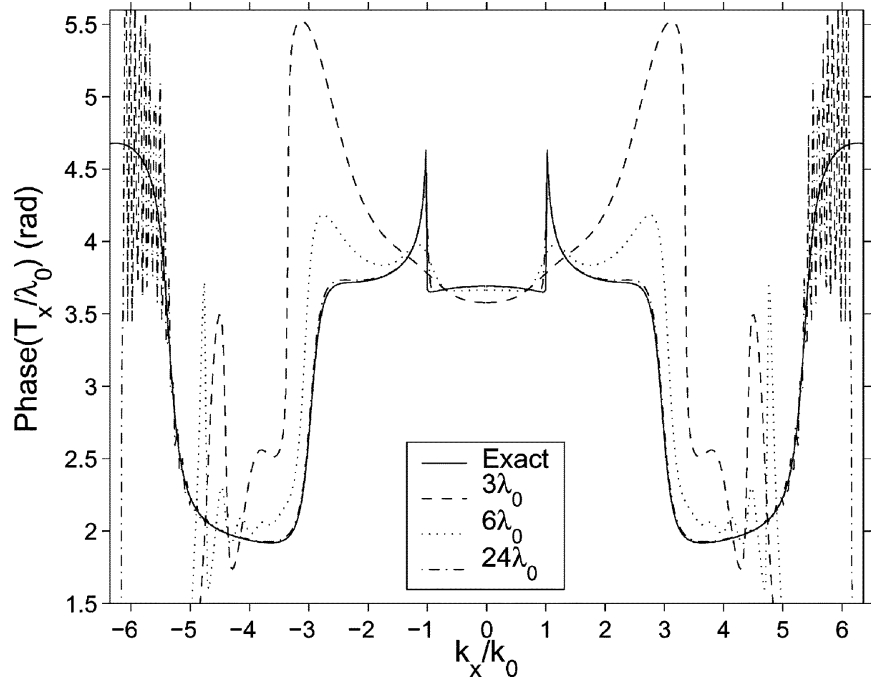

Fig. 15. Phase of the measured $T_{x} / \lambda_{0}$ for $k_{y}=0, z_{a}=0.02 \lambda_{0}, k_{1}^{2}=$ $(8+3 i) k_{0}^{2}$, and $z_{p}=-1.5 \lambda_{1}$. The side length of the square scan plane takes on the values $3 \lambda_{0}, 6 \lambda_{0}$, and $24 \lambda_{0}$.

semianalytical methods. Using the reciprocity theorem, we derived a relation between the plane-wave receiving spectrum and the plane-wave transmitting spectrum for a reciprocal AUT. The relation is exact when it is applied in GPR imaging where the buried objects are described by inhomogeneities in an otherwise homogeneous half space.

Finally, we showed how the plane-wave transmitting and receiving spectra of the AUT can be measured using a probe located in the lower medium. It is necessary to omit the scattering spectra of the AUT/interface system and the probe from the formulation, and hence, multiple interactions between the AUT/interface system and the probe are neglected. This is usually a good approximation because the probe is small, and the lower medium is lossy. Through numerical simulations, we illustrated the concepts associated with determining the minimum scan-plane size and sampling distance for such measurements.

With the plane-wave transmitting and receiving spectra of the AUT available, it is possible to take into account arbitrary antennas in GPR imaging inversion schemes [4], [13]. This task is currently pursued and the result will be published elsewhere.

\section{REFERENCES}

[1] D. M. Kerns, "Plane-wave scattering-matrix theory of antennas and antenna-antenna tnteractions," Nat. Bureau Standards (NIST), Gaithersburg, MD, Tech. Rep. 162, 1981.

[2] T. B. Hansen and A. D. Yaghjian, Plane-Wave Theory of Time-Domain Fields. New York: IEEE Press, 1999.

[3] D. J. Daniels, Surface-Penetrating Radar. London, U.K.: The Inst. Elect. Eng., 1996.

[4] T. B. Hansen and P. Meincke Johansen, "Inversion scheme for ground penetrating radar that takes into account the planar air-soil interface," IEEE Trans. Geosci. Remote Sensing, vol. 38, pp. 496-506, Jan. 2000.

[5] T. B. Hansen and P. Meincke, "Scattering from a buried circular cylinder illuminated by a 3-D source," Radio Sci., vol. 37, pp. 4-1-4-23, Mar. 2002.

[6] D. A. Hill and K. H. Cavcey, "Coupling between two antennas separated by a planar interface," IEEE Trans. Geosci. Remote Sensing, vol. GE-25, pp. 422-431, July 1987.

[7] C. J. Leat, N. V. Shuley, and G. F. Stickley, "Triangular-patch model of bowtie antennas: Validation against Brown and Woodward," Proc. Inst. Elect. Eng.-Microwave Antennas Propagat., vol. 145, pp. 465-470, 1998. 
[8] O. S. Kim, E. Jørgensen, P. Meincke, and O. Breinbjerg, "Higher-order hierarchical discretization scheme for surface integral equations for layered media," IEEE Trans. Geosci. Remote Sensing, vol. 42, pp. 764-772, Apr. 2004.

[9] B. Lampe, K. Holliger, and A. G. Green, "A finite-difference time-domain simulation tool for ground-penetrating radar antennas," Geophysics, vol. 68, pp. 971-987, 2003.

[10] R. W. P. King and G. S. Smith, Antennas in Matter. Cambridge, MA: MIT Press, 1981.

[11] R. V. de Jongh, A. G. Yarovoy, and L. P. Ligthart, "Experimental set-up for measurement of GPR antenna radiation patterns," in Proc. 28th Eur. Microwave Conf., Amsterdam, The Netherlands, Oct. 1998, pp. 539-543.

[12] S. Silver, Microwave Antenna Theory and Design. New York: Dover, 1965.

[13] P. Meincke, "Linear GPR inversion for lossy soil and a planar air-soil interface," IEEE Trans. Geosci. Remote Sensing, vol. 39, pp. 2713-2721, Dec. 2001.

[14] R. E. Collin and F. J. Zucker, Antenna Theory, Part I. New York: McGraw-Hill, 1969.

[15] W. C. Chew, Waves and Fields in Inhomogeneous Media. New York: IEEE Press, 1995.

[16] R. E. Collin, Antennas and Radiowave Propagation. New York: McGraw-Hill, 1985.

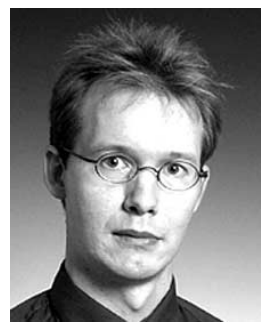

Peter Meincke (S'93-M'96) was born in Roskilde, Denmark, on November 25, 1969. He received the M.S.E.E. and Ph.D. degrees from the Technical University of Denmark (DTU), Lyngby, in 1993 and 1996, respectively.

In the spring and summer of 1995 , he was a Visiting Research Scientist with the Electromagnetics Directorate of Rome Laboratory, Hanscom Air Force Base, MA. In 1997, he was with a Danish cellular phone company, working on theoretical aspects of radio-wave propagation. In the spring and summer of 1998, he was visiting the Center for Electromagnetics Research, Northeastern University, Boston, MA, while holding a Postdoctoral position from DTU. In 1999, he became a staff member of the Department of Electromagnetic Systems, DTU. He is currently an Associate Professor with Ørsted-DTU. His current teaching and research interests include electromagnetic theory, inverse problems, high-frequency and time-domain scattering, antenna theory, and ground-penetrating radars.

Dr. Meincke won the First Prize Award in the 1996 IEEE Antennas and Propagation Society Student Paper Contest in Baltimore, MD, for his paper on uniform physical theory of diffraction equivalent edge currents. Also, he received the 2000 R. W. P. King Paper Award for his paper "Time-Domain Version of the Physical Theory of Diffraction" published in the February 1999 issue of the IEEE TRANSACTIONS ON ANTENNAS AND PROPAGATION.

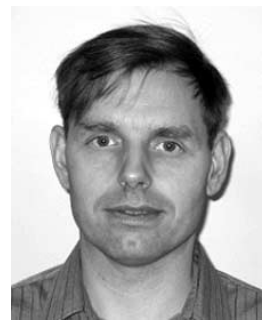

Thorkild B. Hansen (S'91-M'91) was born in Odense, Denmark, in 1965. He received the M.S.E.E. and Ph.D. degrees from the Technical University of Denmark, Lyngby, in 1989 and 1991, respectively.

From 1991 to 1997, he was with the Electromagnetics Directorate of Rome Laboratory (now Air Force Research Laboratory), Hanscom Air Force Base, MA. From 1997 to 2000, he was with Schlumberger Doll Research, Ridgefield, CT, and from 2000 to 2003, he was with Witten Technologies, Incorporated, Boston, MA. He is currently with Seknion, Inc., Boston, a company he cofounded in 2004. He has worked in the areas of electromagnetic theory, low-frequency scattering, high-frequency diffraction, asymptotics, electromagnetic and acoustic wave-field imaging, and inversion. His current research interests include wireless communications. He is coauthor of Plane-Wave Theory of Time-Domain Fields (New York: IEEE Press, 1999).

Dr. Hansen won the 1992 R. W. P. King Prize Paper Award for a paper on corner diffraction published in IEEE TRANSACTIONS ON ANTENNAS AND PROPAGATION. In 1995, he won the IEEE S. A. Schelkunoff Prize Paper Award for two papers on time-domain near-field scanning. He was part of the team that won the 2002 NOVA award for work on ground-penetrating imaging radar. The NOVA Award was instituted by the Construction Innovation Forum to recognize innovations that have proven to be significant advances for the construction industry. He is a member of the Optical Society of America, the American association for the Advancement of science, and URSI Commission B. 